\title{
Role of heat setting and finishing treatment on mechanical properties and hand behavior of stretch fabric
}

\begin{abstract}
This research deals with the effect of heat setting on mechanical properties of the woven fabrics containing spandex. The heat setting treatment given to stretchable fabrics is an important factor in deciding the eventual mechanical comfort properties of the garment. However, finishing treatment also influence fabric mechanical properties as well as hand behavior of the fabric. In this research, the relative potential of both finishing treatment and heat setting process were investigated in order to set appropriate and cost effective finishing route to cotton stretch woven fabric. A total of three woven samples were produced using the same construction parameters. The $100 \%$ cotton warp yarns spun on open end spinning system were used. The weft yarns were the cotton wrapped spandex-core spun yarns. The different processing routes were followed to study the effect of heat setting and finishing process. The fabrics without heat set exhibits higher values for all mechanical properties required for clothing i.e. higher elongation, lesser growth, higher tensile and tear strength, and larger crease recovery angle without any remarkable increase in shrinkage potential both in warp and weft directions. The fabrics without heat set exhibits higher total hand value than heat set treated fabrics for summer wear application. Very limited research studies have been reported with respect to the stretch fabrics. Therefore, a novel attempt has been made in this research work to investigate the role of important processes like heat setting and finishing on the behavioral properties of 'stretch woven fabric'.
\end{abstract}

Volume 6 Issue 5 - 2020

\author{
Sunny Pannu,' Meenakshi Ahirwar, ${ }^{2}$ Rishi \\ Jamdagni, ${ }^{3}$ BK Behera ${ }^{2}$ \\ 'Department of U.I.E.T, Maharshi Dayanand University, India \\ ${ }^{2}$ Department of Textile and Fibre Engineering, Indian Institute of \\ Technology Delhi, India \\ ${ }^{3}$ T.I.T\&S, Bhiwani, India
}

\begin{abstract}
Correspondence: Meenakshi Ahirwar, Ph.D. scholar, Department of Textile and Fiber Engineering, Indian Institute of Technology Delhi, New Delhi, India, Tel 8826077073,
\end{abstract} Email meenakshi736I@gmail.com

Received: July 02, 2020 | Published: September 18, 2020

Keywords: Lycra, stretch woven fabric, heat setting, mechanical properties, fabric handle

\section{Introduction}

The major expectations of a wearer in an apparel fabric are a high level of mechanical comfort and good aesthetic properties. In woven fabrics it is even more challenging to get these properties all together with satisfactory performance. But with the advancements in technology and invention of elastomeric fiber like spandex, it is now possible to enhance the mechanical comfort by means of stretchability. ${ }^{1}$ However, this property of stretchability is greatly influenced by processing and finishing processes applied in case of stretchable fabrics as compared to conventional fabrics. During this stage, the combined effect of water, finishing agents, high temperature and mechanical action results in structural changes in the fabric that may appear in terms of changes in fabric thread density, areal density, thickness and dimensions. Fabrics containing spandex are dimensionally unstable compared to the conventional fabrics. ${ }^{2-4}$ This poor dimensional stability and its optimization is a real challenge for the stretch fabric manufacturers. Therefore, these fabrics are subjected to heat setting treatment to get a satisfactory dimensional stability. ${ }^{5}$ Heat setting is a combined process of thermal and mechanical actions to stabilize the dimensions of the fabric on which it is applied. ${ }^{6}$ Heat set process is one of the important factors that determine the desired fabric properties like ultimate width, weight and its stretch and recovery. ${ }^{1,7}$ Beside these properties, the stenter frame for heat setting is considered the most expensive machine used in a dye house or finishing unit. ${ }^{9}$ Thus, desired fabric properties are achieved at a relatively higher cost.

In view of the above facts, this study was conducted with an objective to analyze the role of important processes like heat setting and finishing on the behavioral properties of 'stretch woven fabric'. The main focus is to achieve the modified or improved cost effective and time saving processes along with the required handle value of fabrics (fabric hand) as per the different end uses. After the processing of the fabrics, the effect on important mechanical properties like EPI (ends per inch), PPI (picks per inch), width, shrinkage, recovery, growth, CRA (crease recovery angle), wrinkle, drape and fabric hand were chosen to analyze the different options to process a 'stretch fabric'. The processing methods used were without heat set (WH), Heat set then pretreated (HP) and Pretreated then Heat set (PH) thus became important objective of the analysis.

Fabric hand is a tactual perception of fabric quality that decides selection of fabric for different end uses. A property judged as a function of the feel of the material, its roughness, smoothness, harshness, pliability, thickness, and so on. ${ }^{10}$ Kawabata and Niwa ${ }^{11,12}$ developed a method for the objective evaluation for the measurement of fabric hand of suiting fabrics. It is the fundamental aspect, which determines the success or failure of many new products. It is an integrated index derived from the low-stress mechanical properties like surface friction, roughness, tensile, shear, bending and compression. ${ }^{13}$ Therefore, the hand evaluation of stretch fabrics after the processing methods becomes an important part of the research because the mechanical comfort also referred as fabric hand is the foremost important requirement of the wearer. The main focus areas were the 'Heat set' and 'Wet Processing' that a stretch fabric was subjected to before finalization. The sole objective is to achieve an ideal practical and feasible route to prepare cost effective 'stretch fabric' with adequate comfort and handle values. And this could be done either by changing the route or by modifying the process itself. 


\section{Methodology}

\section{Materials}

The woven fabrics were prepared in a weaving mill under controlled manufacturing conditions. The warp yarn used was $100 \%$ cotton spun on open end spinning system. The weft yarn used was cotton wrapped spandex-core spun yarn. The construction details of fabric samples are given in Table 1 . The air-jet loom was used to produce fabrics with machine speed of 839rpm. To study the effect of heat setting and finishing processes, the fabric sample were divided into three, each following a different processing route and were coded accordingly as given in Table 2 . The eight replicate fabrics were prepared for each of the three finishing treatment. Therefore, total 24 fabrics were produced using the given construction details.

Table I Construction details of fabrics

\begin{tabular}{llllllll}
\hline Process & Warp & Weft & Weave & EPI & PPI & Reed & Reed space (cm) \\
\hline WH, HP, PH & IO's OE Cotton & $\begin{array}{l}\text { I2's spx,70den cotton spandex- } \\
\text { core spun (spandex \% 4.3) }\end{array}$ & 3/I Z Twill & 64 & 54 & $40 / 7$ & 210.6 \\
\hline
\end{tabular}

Table 2 Code for the different fabric samples

\begin{tabular}{ll}
\hline Code & Description \\
\hline WH & Grey fabric Without Heat set \\
HP & Grey fabric Heat set then Pretreated \\
PH & Grey fabric Pretreated then Heat set \\
\hline
\end{tabular}

\section{Methods}

\section{Process sequence}

The grey fabrics were processed according to the three different processing routes with the standard process parameters used for commercial production as given in Table 3 . The temperature used for heat setting was $190^{\circ} \mathrm{C}$ with speed $55 \mathrm{~m} / \mathrm{min}$. The temperatures for pretreatment and mercerization were $98^{\circ} \mathrm{C}$ and $60^{\circ} \mathrm{C}$ respectively with $90 \mathrm{~m} / \mathrm{min}$ speled.

Table 3 Process route for different samples

\begin{tabular}{ll}
\hline Sample & Process route \\
\hline WH & Grey fabric $\rightarrow$ Grey Wash $\rightarrow$ Pretreatment $\rightarrow$ Mercerization $\rightarrow$ Peach finish $\rightarrow$ Wash $\rightarrow$ Dry Pad $\rightarrow$ Pad Steam $\rightarrow$ Finishing $\rightarrow$ Drying \\
HP & Grey fabric $\rightarrow$ Grey Wash $\rightarrow$ Heat set $\rightarrow$ Pretreatment $\rightarrow$ Mercerization $\rightarrow$ Peach finish $\rightarrow$ Wash $\rightarrow$ Dry Pad $\rightarrow$ Pad Steam $\rightarrow$ Finishing $\rightarrow$ \\
& Drying \\
PH & $\begin{array}{l}\text { Grey fabric } \rightarrow \text { Grey Wash } \rightarrow \text { Pretreatment } \rightarrow \text { Mercerization } \rightarrow \text { Heat Set } \rightarrow \text { Peach finish } \rightarrow \text { Wash } \rightarrow \text { Dry Pad } \rightarrow \text { Pad Steam } \rightarrow \text { Finishing } \rightarrow \\
\text { Drying }\end{array}$
\end{tabular}

\section{Fabric characterization}

The fabrics were evaluated for important mechanical properties like fabric count, fabric width, fabric shrinkage, fabric tensile and tear strength and some aesthetic properties like crease recovery angle, wrinkle recovery and drape. The fabric samples were tested according standard test methods as given in Table 4. An average of five observations was taken for each sample.

Determination of dimensional properties: The properties like EPI, PPI, GSM, and thickness of the fabrics were determined after the different processing methods. All the tests were carried out as per the standards given in Table 4.

Table 4 Test standards for fabric properties evaluation

\begin{tabular}{lll}
\hline Parameters & Unit & Test method \\
\hline Finish EPI \& PPI & - & ASTM D 3775-2003 \\
Finish width & $\mathrm{cm}$ & ASTM D 3774-96 \\
Weight & $\mathrm{g} / \mathrm{m}^{2}$ & ASTM D 3776-2002 \\
Shrinkage (Warp \&Weft) & $\%$ & AATCC-I35 \\
Tear strength (Warp \&Weft) & $\mathrm{N}$ & ASTM D- I424 \\
Tensile strength (Warp \&Weft) & $\mathrm{N}$ & ASTM-5034 \\
Elongation (initial \% A/F 3 wash) & $\%$ & ASTM-3 I07 \\
Growth (initial \% A/F 3 wash) & $\%$ & ASTM-3 I07 \\
Crease recovery angle & Degree & AATCC-66-2014 \\
Wrinkle recovery & - & AATCC- I28 (Appearance method) \\
Drape & $\%$ & Cusick Drapemeter \\
Total Hand Value & - & Kawabata Evaluation System \\
\hline
\end{tabular}


Determination of stretch properties: According to ASTM standard D3107-07, fabric's stretch properties include 'fabric stretch under a specified tension', 'fabric growth after a specified tension', 'fabric growth after stretching to a specified extension', and 'fabric recovery'. Fabric stretch is evaluated as the measured resulting length difference between the benchmarks on the test specimen before and under the applied tension after a specified time. Fabric growth is evaluated as the measured resulting length difference between the benchmarks on the test specimen before the applied tension and after relaxation for a specified time. Fabric recovery is evaluated as the percentage of fabric growth recovered with respect to the fabric growth after applied tension.

The three pairs of samples with dimension $65 \mathrm{~mm} \times 560 \mathrm{~mm}$ were taken with long dimension parallel to the test direction. Benchmarks were placed on each sample $250 \mathrm{~mm}$ apart. The test specimen was clamped in the testing apparatus with a hanging load of $1.8 \mathrm{~kg}$ for stretching. The distance between the benchmarks was measured after $10 \mathrm{sec}$. The load and lower clamp of apparatus were removed to allow the sample to recover.

Then the fabric stretching (eq. i), fabric growth (eq. ii) and elastic recovery (eq. iii) of the fabrics were calculated:

Fabric stretching $(\%)=100 \times(B-A) / A(i)$

Fabric growth $(\%)=100 \times(\mathrm{C}-\mathrm{A}) / \mathrm{A}$ (ii)

Elastic recovery $(\%)=100 \times(B-C) /(B-A)($ iii)

Where, A-the distance between the upper and bottom benchmark on the test specimen $(250 \mathrm{~mm}), \mathrm{B}-\mathrm{The}$ distance between the upper and bottom benchmark after $10 \mathrm{sec}$ of loading the test specimen $(\mathrm{mm})$ and C-The distance between the upper and bottom benchmark after 1-hour relaxation

Determination of crease recovery angle: The dry crease recovery angle was measured for all the samples by using Shirley crease recovery tester. A $40 \times 15 \mathrm{~mm}$ specimen size was used. Each test specimen was folded face to face and creased under $2 \mathrm{~kg}$ weight for $1 \mathrm{~min}$ and then allowed to recover from creasing for $1 \mathrm{~min}$. For each fabric, six specimens were cut in each warp and weft direction. Out of six, three specimen were tested with folding face to face of sample and three with folding back to back creasing. Finally, the average of six values of warp-wise and weft-wise crease recovery angle was measured. ${ }^{14}$ IS 4681-1968 standard was used for this purpose.

Determination of tensile properties: The tensile strength test of fabric samples was carried out according to the ASTM D 5035-06. The grab test evaluates breaking force as the maximum force applied to the fabric sample to rupture it. Specimen size of $100 \mathrm{mmx} 150 \mathrm{~mm}$ was taken with long direction parallel to direction of test. The $75 \mathrm{~mm}$ gauge length is fixed in apparatus. The samples were tested with a loading rate of $300 \mathrm{~mm} / \mathrm{min}$. Total five samples were tested each for warp and weft directions.

Determination of tear strength: The fabric tear strength was measured by using falling-pendulum type (Elmendorf) tearing tester according to the test procedure of ASTM D1424. The sample size taken was $100 * 63 \mathrm{~mm}$. Tear force used was $33.77 \mathrm{~N}$. The unit of tear strength was Newton. Five reading were taken in warp direction and five in weft direction. The average of warp and weft samples was taken.

Determination of fabric drape: The drape for each fabric sample was evaluated in terms of drape coefficient by using Cusick drapemeter with a circular test specimen of diameter $30 \mathrm{~cm}$. Two specimens were tested for each fabric sample and from both face and back sides. An average of four readings was taken.

Determination of wrinkle recovery: The wrinkle recovery of fabrics was determined by using appearance method according to AATCC 128. According to this method, a large number of random wrinkles were induced intentionally in the test sample under standard atmospheric conditions. A standard device was used to wrinkle fabric using a load of 3500 grams for $20 \mathrm{~min}$. After wrinkling, the test specimen was re-conditioned for 24 hour after load removal and rated for appearance by three trained observers. The rating was given to samples by comparing them with AATCC wrinkle recovery replicas standard samples. The sample size was $6 * 11$ inch.

Determination of fabric hand: Kawabata Evaluation System (KES) was used for measuring the sixteen low stress mechanical properties of the fabrics. Tensile and shear, bending, compression, and surface properties were evaluated using KES-FB1, KES-FB2, KES-FB3, and KES-FB4 instruments respectively. ${ }^{13}$ The fabric sample size was $20 * 20 \mathrm{~cm}$. For each sample ten readings were measured both in wrap and weft directions. Furthermore, the Kawabata set of equations were used to evaluate the primary hand values like stiffness, smoothness, crispness etc. and the total hand values were estimated using standard hand equation prescribed by Kawabata for summer and winter suiting garments. The primary hand value and the total hand value obtained were in a scale of $0-10$ and $0-5$ respectively.

\section{Results and discussions}

\section{Effect of post weaving processes on dimensional properties of stretch fabrics}

The fabrics without heat-set, heat-set then pretreated and pretreated then heat-set were tested for EPI, PPI, GSM, width, shrinkage, growth and recovery. The results are shown in Figures 1-4. The Figure 1 shows that there is no significant difference in EPI among the three samples due to the three different processing approaches. Though, the grey washing results in an increase in EPI in general because of the relaxation of the temporary stresses in the yarns imposed during weaving. ${ }^{15}$ There is an overall increase in EPI of fabrics due to different finishes applied on the fabric.

The Figure 2 shows a falling trend in PPI for the fabrics that has undergone heat setting treatment and an increase in PPI was observed for fabric processed without heat set. Among the three methods the heat set then pretreat shows comparatively more decrease in PPI.

The Figure 3 shows effect of different processes on fabric areal density GSM and a significant difference can be observed due to different processing route. The without heat set sample shows comparatively higher values for areal density followed by heat set then pretreated and then pretreated then heat set sample. The literature advocates the generation of higher retraction force by weft yarns containing spandex. This results in off loom fabric contraction along the weft direction. ${ }^{16}$

The Figure 4 shows a very little difference in values of fabric width due to different processing route. The without heat set sample shows comparatively higher decrease in width then heat set then pretreated and pretreated then heat set samples respectively. The two samples undergone heat setting show almost same values of final width. During processing, the fibers contained in yarn tend to relax and finally after heat set, the spandex filament is fixed at more relaxed stage thus leaving a fabric with less potential to shrink further widthwise. ${ }^{2,17}$ 


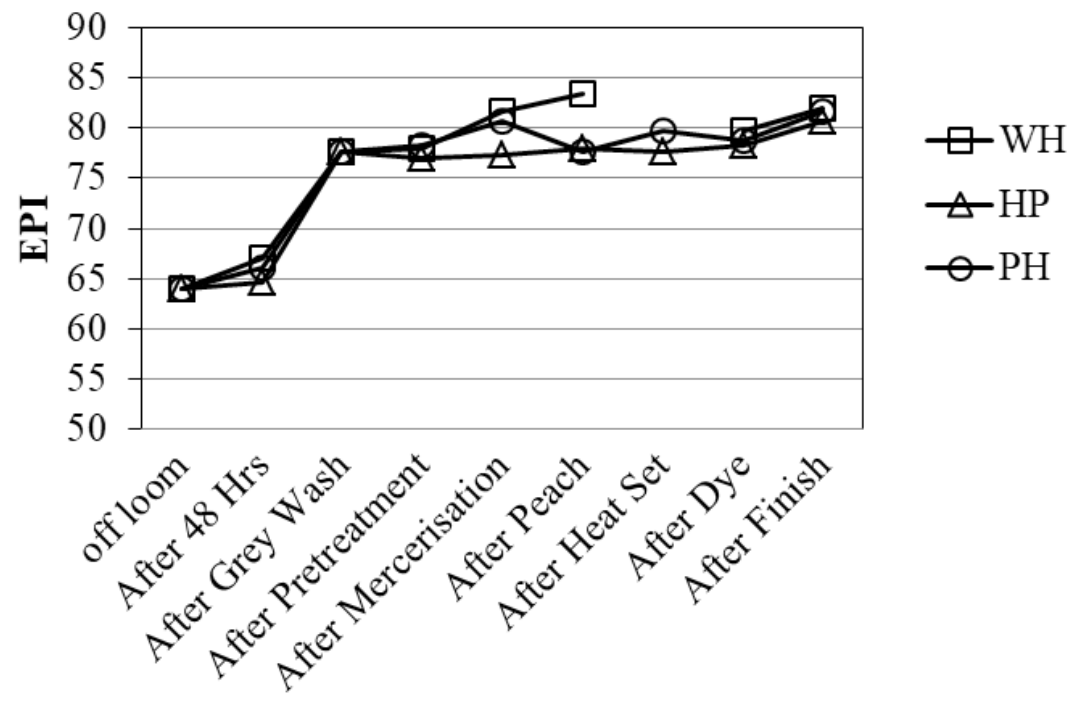

Figure I Effect of different processes on ends per inch (EPI).

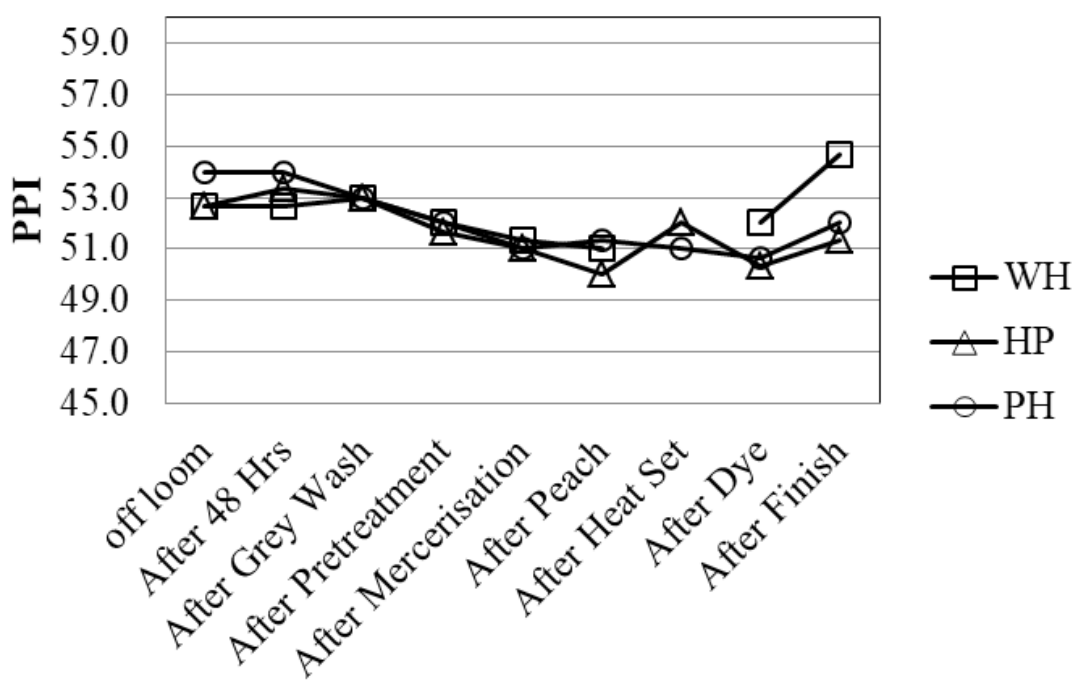

Figure 2 Effect of different processes on picks per inch (PPI).

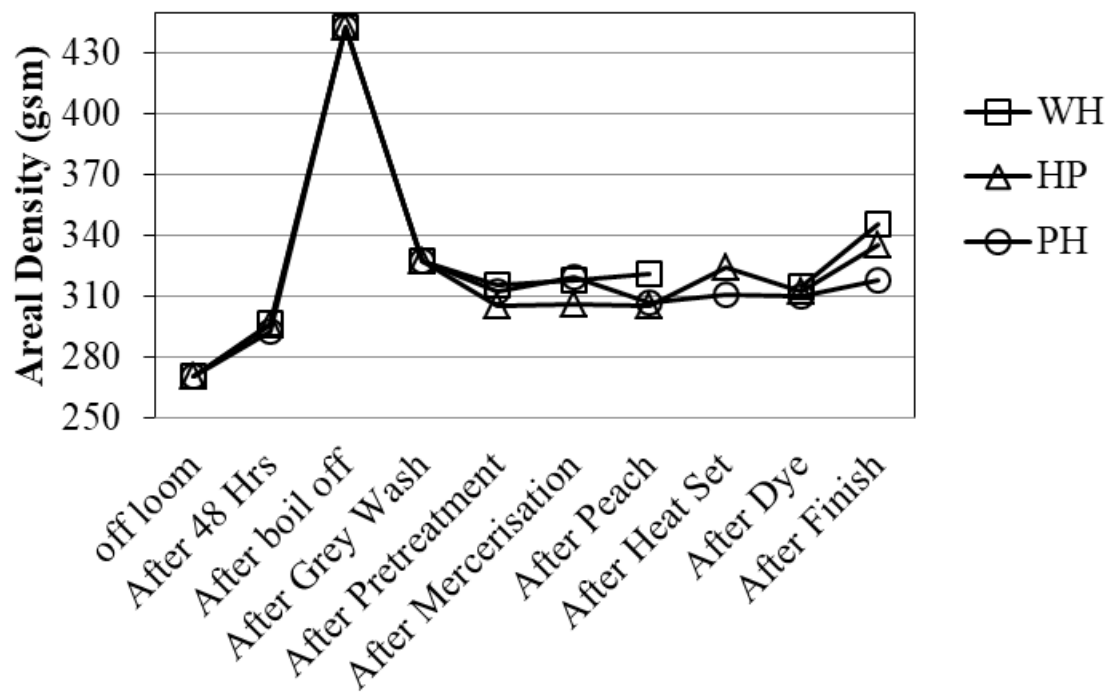

Figure 3 Effect of different processes on Areal Density (GSM). 


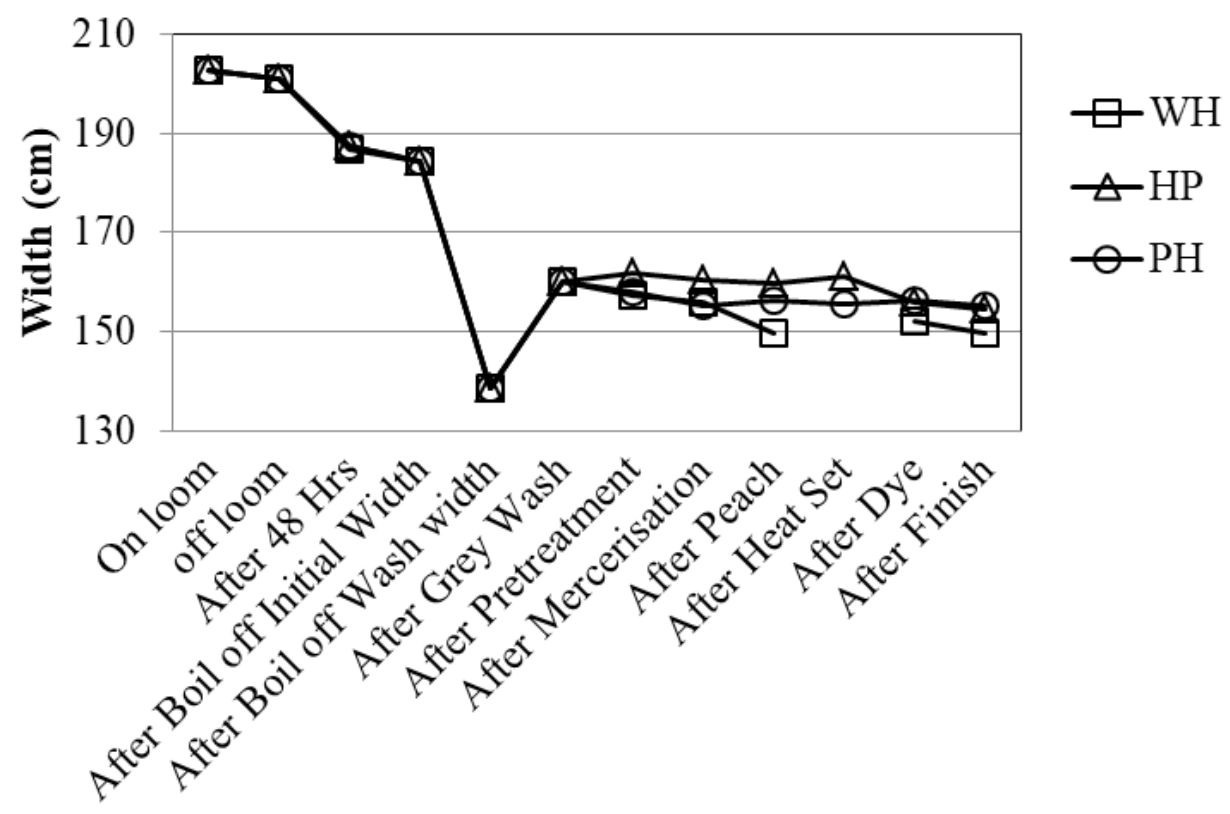

Figure 4 Effect of different processes on width.

\section{Effect of post weaving processes on stretch properties of woven stretch fabrics}

In case of a spandex based stretch fabric, with every post weaving process, fabric undergoes perceptible dimensional changes. These changes become significantly important particularly when finishing process includes heat treatment. The Figure 5 shows an overall increase in elongation due to finishing processes. There is maximum elongation in without heat set sample followed by heat set then pretreated sample and finally pretreated then heat set sample. This was because of heat that fixed the spandex filament in a more relaxed stage. However, a little increase is also represented in elongation values even after heat setting. This behavior can be attributed to successive shrinkage of non-elastic cotton sheath fibers in core spun yarns during subsequent wet processing. ${ }^{3}$ The Figure 6 shows an overall increase in growth. The without heat set sample shows least growth followed by heat set then pretreated and pretreated then heat set samples. The results indicated that the heat treatment during heat setting limits the elasticity of spandex yarn to some extent results in higher growth in such samples. ${ }^{18}$

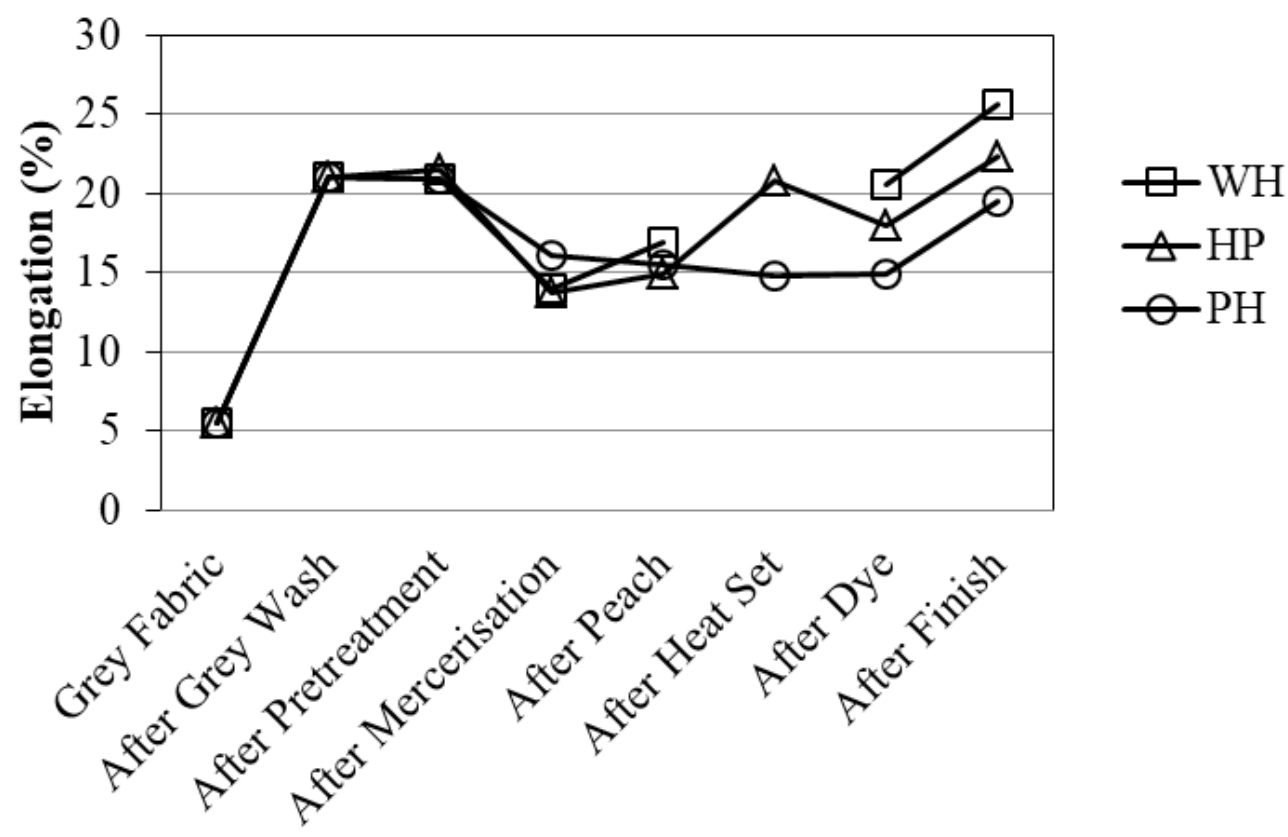

Figure 5 Effect of different processes on elongation. 


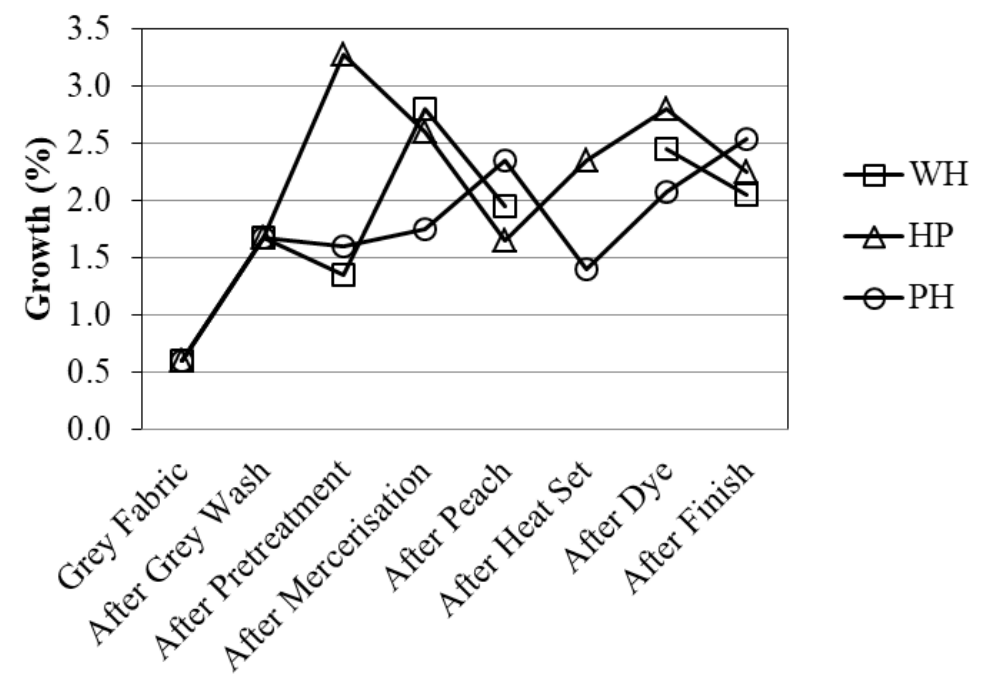

Figure 6 Effect of different processes on growth.

Figure 7 and Figure 8 depicts the shrinkage behavior warp wise and weft wise. There is an overall reduction in shrinkage percentage in due processes of pretreatment, dyeing and final finish. The three object samples show similar trend warp wise but there is an early control of weft shrinkage in case of heat set then pretreated sample than other two. After final finish, the three samples show comparatively same values of shrinkage for warp and weft which means that different processing route has no significant influence on fabric shrinkage.

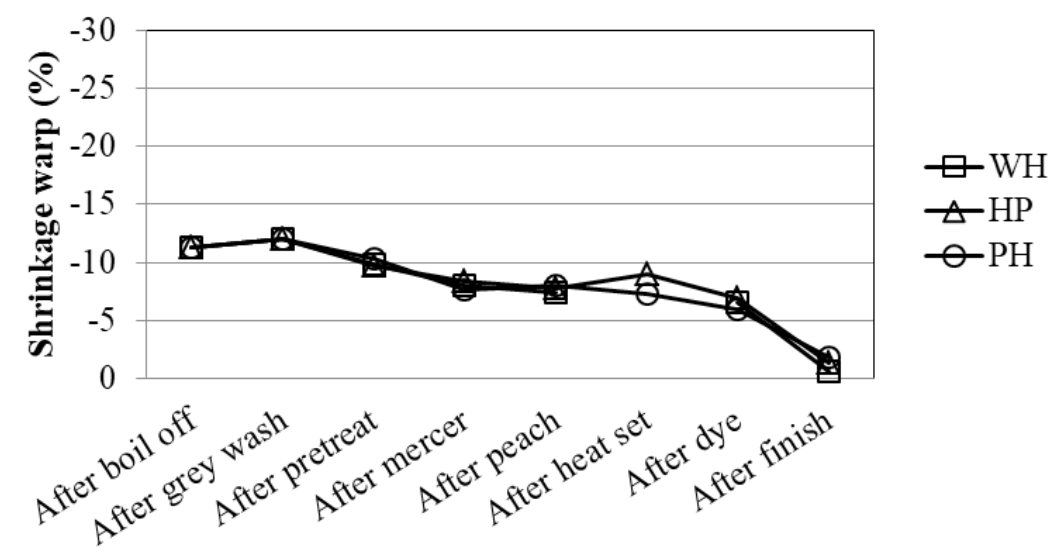

Figure 7 Effect of different processes on warp shrinkage.

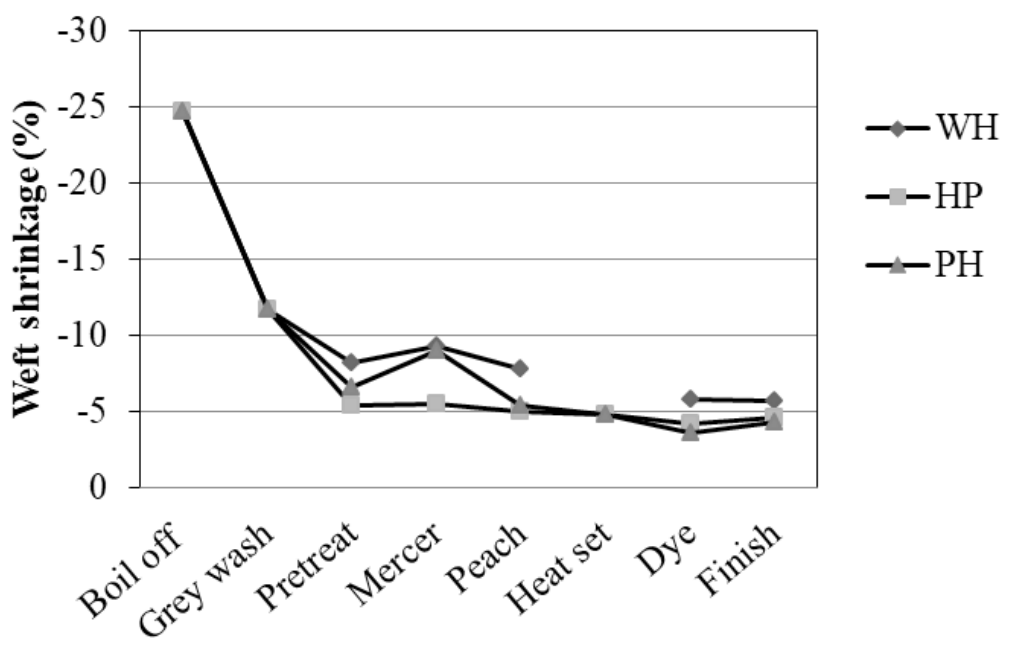

Figure 8 Effect of different processes on weft shrinkage. 


\section{Effect of heat setting and various finishing treatments on mechanical properties of woven stretch fabrics}

In Figure 9 and Figure 10, the tensile and tearing properties have been analyzed after mercerization and final finish. It was observed that the three samples do not show any significant difference in tensile strength values due to difference in processing route followed up to mercerization. But after final finish, a difference can be noticed among three samples. The tensile strength values show a decreasing trend in order of without heat set, pretreated then heat set and heat set then pretreated respectively. Similar trend was seen in tensile strength after finish in weft direction.

The final finish has a positive impact on tearing strength of warp and weft yarns. Among the samples, the tearing strength shows a reducing trend in order of without heat set, heat set then pretreated and pretreated then heat set.

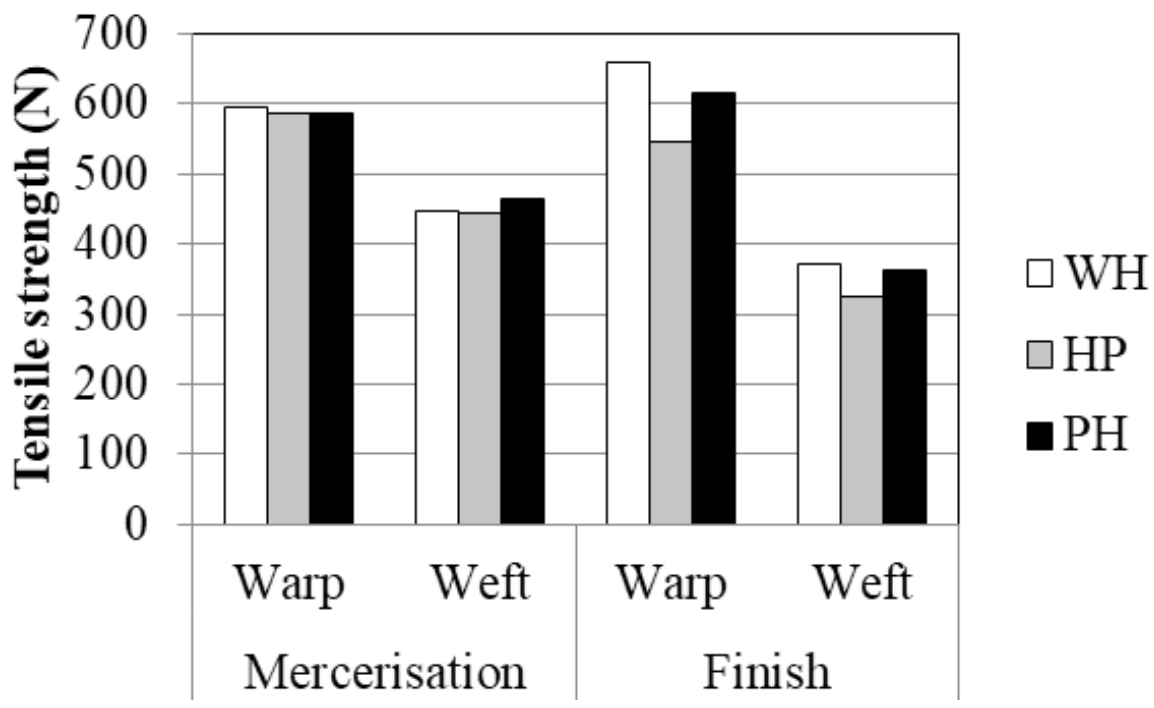

Figure 9 Effect of different processes on tensile strength (warp and weft wise).

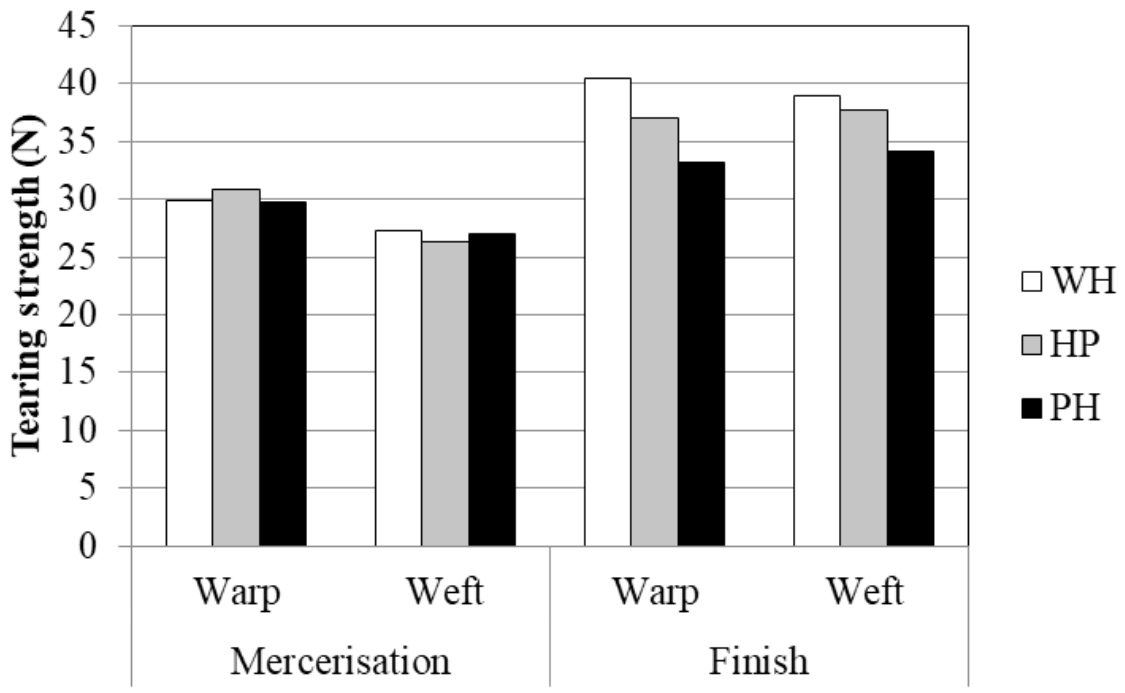

Figure 10 Effect of different processes on tearing strength (warp and weft wise).

\section{Effect of finishing treatments on aesthetic properties of woven stretch fabrics}

The Figure 11 shows the crease recovery angle for face and back in warp and weft directions for three samples. The crease recovery angle was higher in weft direction than in warp because of the incorporation of spandex filament in weft yarns that results in a more flexible yarn. There was an improvement in crease recovery angle in all the three samples after finish. However, without heat set sample shows the most significant increase in crease recovery angle. The results of wrinkle recovery test and drape are shown in Table 5. The rating for wrinkle recovery shows no significant difference among three object samples. In case of drape, the drape coefficient was higher for without heat set sample followed by heat set then pretreated and pretreated then heat set sample. 


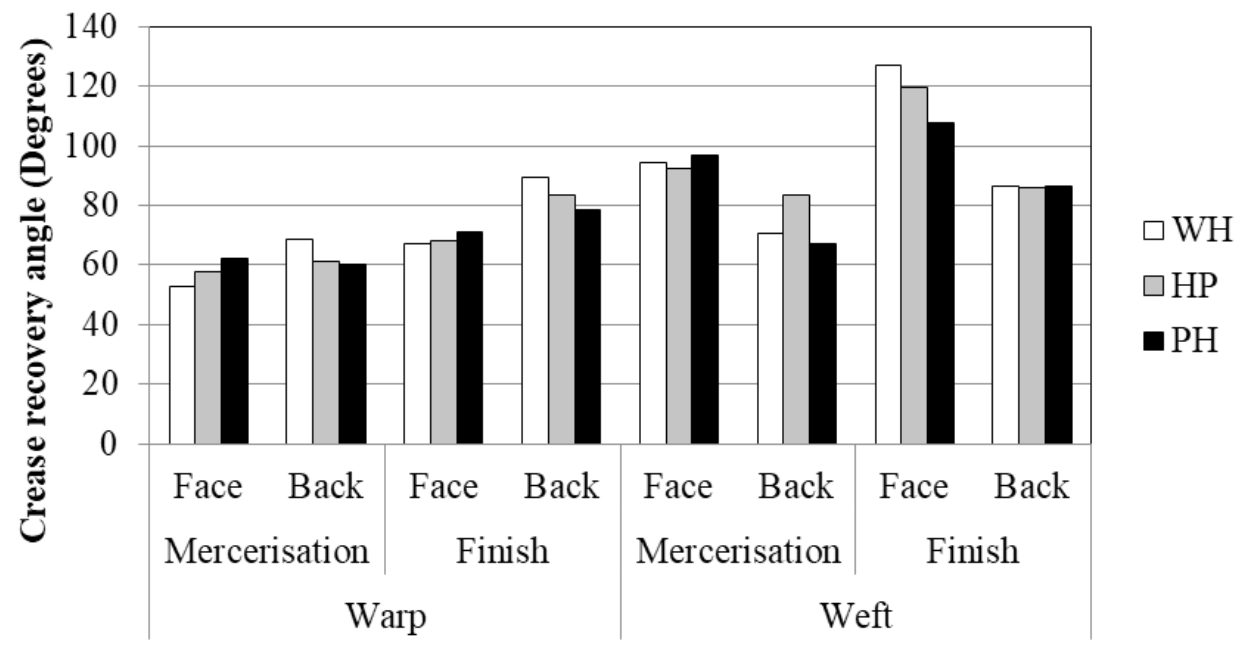

Figure II Effect of different processes on CRA (warp and weft wise).

Table 5 Test results for wrinkle recovery and drape

\begin{tabular}{lllll}
\hline \multirow{2}{*}{ Code } & \multicolumn{2}{l}{ Wrinkle recovery rating } & \\
\cline { 2 - 4 } & Observer A & Observer B & Observer C & \\
WH & 2 & 2 & 3 & 78.71 \\
& 3 & 2 & 3 & \\
& 3 & 3 & 3 & 70.33 \\
HP & 3 & 3 & 3 & \\
& 2 & 3 & 2 & \\
& 1 & 2 & 2 & 60.24 \\
PH & 3 & 1 & 2 & \\
& 3 & 2 & 2 & \\
\hline
\end{tabular}

\section{Effect of heat treatment on hand behavior of woven stretch fabrics}

Hand is a tactual perception of fabric quality. It is an indicative of mechanical comfort of the fabric which is derived from low stress mechanical properties of the fabric. Table 6 shows the results of total hand value obtained from KES evaluation of the three object samples in view of the summer and winter suiting applications. The three samples were found unsuitable for summer suiting and fits well for winters. The decreasing order of THV values is follows as without heat set, pretreated then heat set and heat set then pretreated respectively. The findings of hand value also indicate that there is no remarkable influence of heat setting on handle of the stretch woven fabric. The results clearly reveal that heat setting does not affect the mechanical properties of the fabric at low stress level. This is probably due to the fact that the synthetic spandex component in the yarn is very low which only contribute to stretchability substantially.
Table 6 Results of KES evaluation

\begin{tabular}{llll}
\hline \multirow{2}{*}{ Code } & Code description & \multicolumn{2}{l}{ THV } \\
\cline { 3 - 4 } & & Summer & Winter \\
\hline WH & Without heat set & 0.86 & 3.78 \\
HP & Heat set then pretreated & 1.54 & 3.4 \\
PH & Pretreated then heat set & 1.62 & 3.78 \\
\hline
\end{tabular}

\section{Conclusion}

The study of the research shows comparative behavior of three processing and finishing routes to produce spandex fabric namely without heat set, first heat set then pre-treated and first pre-treated then heat set. It can be clearly observed in the results that finishing has a great influence on the fabric properties as it causes the fibers and yarns of fabric to relax from the induced tensions and stresses during 
weaving. As a result, the fabric contracts and overall decrease can be seen in fabric shrinkage both warp and weft wise. This ultimately affects the elongation and growth of the final finished fabric.

The fabric sample without heat set exhibits higher values for all important mechanical properties required for clothing i.e. higher elongation, lesser growth, higher tensile and tear strength, larger crease recovery angle and higher drape coefficient and all these without any remarkable increase in shrinkage potential both in warp and weft direction. Moreover and most importantly, the sample without heat set exhibits a total hand value almost equal to the samples that undergone heat set treatment.

\section{Acknowledgments}

None.

\section{Funding}

None.

\section{Conflicts of interest}

The authors have no conflicts of interest regarding the publication of this paper.

\section{References}

1. Mathur K, Elnashar EA, Hauser P, et al. Stretch potential of woven fabrics containing spandex. 5th International Conference of Textile Research Division NRC, Cairo, Egypt; 2008:1-19.

2. Ozdil N. Stretch and bagging properties of denim fabrics containing different rates of elastane. FIBRES Text East Eur. 2008;16:63-67.

3. El-Ghezal S, Babay A, Dhouib S, et al. Study of the impact of elastane's ratio and finishing process on the mechanical properties of stretch denim. J Text Inst. 2009;100(3):245-253.

4. Gorjanc DS, Bizjak M. Impact of pre-finishing process on comfort characteristics of stretchable cotton fabric. J Eng Fiber Fabr. 2015;10:5768.
5. Nazir A, Hussain T, Rehman A, et al. Modelling heat-setting of cotton/ elastane knitted fabrics for optimum dimensional stability. J Text Appar Technol Manag. 2015;9:1-12.

6. Besler N, Gloy Y, Gries T. Analysis of the heat setting process. 48th Conference of the International Federation of Knitting Technologists (IFKT). 2016.

7. Varghese N, Thilagavathi G. Development of woven stretch fabrics and analysis on handle, stretch, and pressure comfort. J Text Inst. 2015;106:242-252.

8. Mansoor T, Siddique HF, Ali A, et al. Wrinkle free plaited knitted fabrics without pre- heat setting. J Text Inst. 2018;109:307-311.

9. Choudhary AKR. Introduction to finishing. Principles of Textile Finishing. Woodhead publishing Series in Textiles; 2017:1-19.

10. Schwartz ER. Technical evaluation of finishing treatments. Am Dyest Report. 1939;28:138.

11. Kawabata S, Niwa M. Chapter 10, objective measurement of fabric hand Mordern Textile Characterization Methods; 1996.

12. Kawabata S. The standardization and analysis of hand evaluation. 1980.

13. Majumdar A, Pol SB. Low stress mechanical properties of fabrics woven from bamboo viscose blended yarns. Fibers Polym. 2014;15:1985-1991.

14. Subramanian S, Phalgumani GR, Manjunatha BR, et al. Assessment of crease recovery values of textile fabrics by different instruments. $1983 ; 8: 16-22$.

15. Kan CW, Yuen CWM. Evaluation of the performance of stretch denim fabric under the effect of repeated home laundering processes. Int J Fash Des Technol Educ. 2009;2(2-3):71-79.

16. Qadir B, Hussain T, Malik M. Effect of elastane denier and draft ratio of core-spun cotton weft yarns on the mechanical properties of woven fabrics. J Eng Fiber Fabr. 2014;9:23-31.

17. Mourad MM, Elshakankery MH, Almetwally AA. Physical and stretch properties of woven cotton fabrics containing different rates of spandex. $J$ Am Sci. 2012;8:567-572.

18. Ibrahim M. Mechanisms of stretch development in fabrics containing spandex yarns. Text Res J. 1966:696-706. 